\title{
Non-traumatic Hematomyelia: A Rare Finding in Clinical Practice
}

\author{
Eva Claro $^{1}$, Alexandra Dias ${ }^{1}$, Geetha Girithari $^{1}$, Ana Massano $^{2}$, Maria Aurora Duarte $^{1}$ \\ ${ }^{1}$ Department of Internal Medicine, Centro Hospitalar do Meio Tejo, EPE, Tomar, Portugal \\ ${ }^{2}$ Department of Neurology, Centro Hospitalar do Meio Tejo, EPE, Abrantes, Portugal
}

Received: 19/09/2018

Accepted: 25/09/2018

Published: 16/10/2018

How to cite this article: Claro E, Dias A Girithari G, Massano A, Duarte MA. Non-traumatic hematomyelia: a rare finding in clinical practice. EJCRIM 2018;5: doi:10.12890/2018_000961.

Conflicts of Interests: The Authors declare that there are no competing interests.

This article is licensed under a Commons Attribution Non-Commercial 4.0 License

\section{ABSTRACT}

Non-traumatic haematomyelia is a rare finding of acute onset, which in most cases is the result of arteriovenous malformations (AVM), tumours, coagulation disorders or autoimmune conditions, but may also be secondary to treatment with anticoagulants and radiotherapy. We present the case of a 58-year-old woman with sudden onset cervical pain, followed by asymmetric diminution of strength in the upper limbs with reduced pain sensitivity. The diagnosis of AVM at the C7 and D1 levels was made following cervico-dorsal magnetic resonance imaging and angiography. Treatment was embolization with immediate isolation of the AVM.

\section{LEARNING POINTS}

- Central nervous system lesions (including subarachnoid haemorrhage) can be located in the spine as well as the brain.

- Subarachnoid haemorrhage can be diagnosed through examination of the cerebrospinal fluid, especially when the CT scan is normal in patients with typical signs of this condition (acute neurological deficits with pain and signs of meningeal irritation like fever or neck rigidity).

- Non-traumatic haematomyelia is a rare finding.

\section{KEYWORDS}

Haematomyelia, arteriovenous malformations, spinal cord, magnetic resonance imaging, angiography

\section{INTRODUCTION}

Haematomyelia is an infrequent finding, and mostly secondary to spinal cord trauma. The main aetiologies of the even rarer non-traumatic haematomyelia are arteriovenous malformations and cavernomas, followed by complications of anticoagulant therapy (warfarin), congenital anomalies of coagulation (von Willebrand's disease, factor VII, VIII or IX deficiency), primary marrow tumours (ependymoma, fibrillar astrocytomas and primary melanoma of the central nervous system) or metastasis (lung, breast and renal cell carcinoma), and less frequently, are secondary to radiotherapy ${ }^{[1]}$.

Clinically, non-traumatic haematomyelia can have an acute, subacute or chronic presentation, characterized by intense, sudden pain in the rachis with or without radicular radiation below the level of the lesion. Fasciculations or areflexia have been observed in the corresponding myotomes $^{[2]}$.

We describe a clinical case in which the presentation of haematomyelia, because it is rare, presented a challenging and unusual differential diagnosis. 


\section{CASE REPORT}

A 58-year-old woman with no relevant medical history was admitted to the emergency department for fasciculations in all four limbs, with reduced muscle strength on the left side of the body, preceded by intense posterior neck pain. At hospital admission we observed a fever of $38^{\circ} \mathrm{C}$ and atrial fibrillation with a rapid ventricular response. The neurological examination showed asymmetric brachial paralysis (grade 2 on the left arm and grade 4 on the right arm) with predominance in the extensor groups, without osteotendinous reflexes in the left upper limb, with hypoaesthesia in the C4-C7 territory on the left and T2-T10 on the right, and without sphincter incontinence. No markers of inflammation or raised cardiac enzyme levels were detected. A cranio-cervical computerized tomography (CT) scan with and without contrast did not show any changes. However, xantochromatic cerebrospinal fluid (CSF; Fig. 1) obtained by a subsequent lumbar puncture without increased pressure, revealed $77 / \mathrm{mm}^{3}$ erythrocytes, raised protein levels (621 mg/dl, normal upper limit $45 \mathrm{~mm}^{3}$ ) and 129/mm leukocytes. Blood and CSF samples sent for culture and for screening for viruses and fungi were negative.

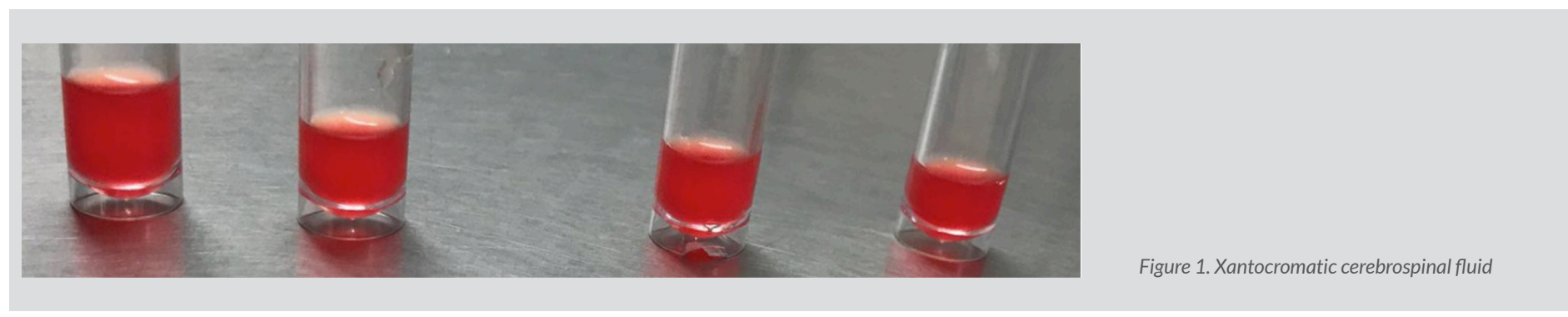

A cervico-dorsal MRI identified intramedullary lesions at the level of C7 and D1 with heterogeneous signal evolution in all weights, hyper/ hypointensity without appreciable signal enhancement after gadolinium, with irregular contours and causing spinal expansion. An extensive area of intramedullary vasogenic oedema with progression above C1 and below D2 was also seen. These alterations were compatible with haematomyelia (in the probable context of rupture of a spinal-medullary vascular malformation) with apparent coexistence of an extramedullary intradural haemorrhagic component to the left.

Angiography performed to better characterize the lesion demonstrated an intracanal arteriovenous malformation (AVM) with afferency of the deep cervical artery, and a branch of the costocervical trunk at the C7 level, apparently intramedullary in anterior view. During the procedure, the AVM was embolized with selective cauterization of the afferent branch. The patient is currently in a rehabilitation program and 2 months after her presentation still has abdominal and right arm hypoaesthesia and grade 3/5 right hemiparalysis, but can walk long distances with the aid of a walker.

\section{DISCUSSION}

The sudden onset of neurological symptoms associated with paroxysmal atrial fibrillation in this patient suggested cerebrovascular pathology due to a cardiac embolism. As the patient also had fever with cervical pain without an obvious infectious focus, we had to rule out infection of the central nervous system. We therefore performed a CT scan followed by a lumbar puncture, which led us to the diagnosis of haematomyelia. The clinical history ruled out various aetiologies, but AVM, tumours and metastasis were possibilities. The MRI confirmed the presence of subarachnoid blood, leading to angiography to better characterize the aetiology.

An intramedullary spinal AVM is usually supplied by drainage from medullary arteries and veins. When a haemorrhage occurs, myelopathy is the result of a mass effect, ischaemia or haemorrhage into the cord. In some patients, this can also evolve to subarachnoid haemorrhage. In our case, examination of the CSF revealed xanthochromia. After contact with CSF, haemoglobin breaks down to oxyhaemoglobin, which colours the CSF pink ${ }^{[3]}$. Therefore, CSF examination is useful in the diagnosis of subarachnoid haemorrhage, especially when the CT scan is normal.

Vascular malformations of bone marrow are the most frequent causes of haematomyelia occurring without a history of trauma. These malformations include AVM, cavernomas, capillary telangiectasias and venous angiomas. A diagnosis of haematomyelia indicates the presence of a spinal syndrome with severe pain in the region of the spine with or without radicular radiation. Therefore, a careful clinical history and a detailed physical examination can provide clues to the diagnosis.

However, the presentation of spinal cord haemorrhage depends on the location and severity of the lesion, which makes the diagnosis challenging. Imaging tests are of paramount importance for elucidating the aetiology of the condition, with MRI being the examination of choice, together with angiography ${ }^{[4]}$. 
As AVM are unusual entities, there is no consensus concerning their management, but it is important to avoid the recurrence of bleeding. Some recommend surgical treatment as soon as possible if there is neurological deterioration. However, if neurological symptoms are stable, close monitoring is recommended with intervention (endovascular embolization or surgical clipping) carried out only when the lesion has been properly characterized. Endovascular embolization is preferable to surgery, since it has less risk of complications ${ }^{[5]}$. While the patient is waiting for surgical treatment, progression of the lesion must be avoided by suspension of anticoagulants or anticlotting agents, blood pressure control and resting with the head of the bed raised by 30 degrees.

\section{CONCLUSION}

Haematomyelia has many clinical manifestations depending on the location of the lesion, but always has the common feature of sudden onset of acute pain in the spine with radicular radiation, which can mimic cerebrovascular disease. However, clinical examination is not sufficient to diagnose this condition, as in this case, where CSF obtained by lumbar puncture and coloured pink by blood was key for diagnostic progress. The imaging test allowed us to reach a definitive diagnosis. Treatment to eliminate the cause of bleeding should be initiated as soon as possible to preserve and restore neurological function. Prognosis is closely related to the degree of neurological degradation. Haematomyelia can often lead to permanent disability which can be decreased with quick diagnosis and treatment based on clinical history and physical examination to determine the best imaging method, taking into account that the CNS lesion can be located outside the brain.

\section{REFERENCES}

1. Shaban A, Moritani T, Al Kasab S, Sheharyar A, Limaye KS, Adams HP Jr. Spinal cord hemorrhage. J Stroke Cardiovasc Dis 2018;27:1435-1446

2. Wang H-F, Wang D-M. Exclusively epidural spinal arteriovenous malformation: a short review. Neuropsychiatry (London) 2017;7:897-905.

3. Lufang Y, Gordon B, Gordon H. Detection of xanthochromia in cerebrospinal fluid by spectrophotometric scan. Am J Clin Pathol 2014;142(Suppl 1):A02.

4. Leep Hunderfund AN, Wijdicks EF. Intramedullary spinal cord hemorrhage (hematomyelia). Rev Neurol Dis 2009;6:E54-61.

5. Choi BS, Lee S. Idiopathic spontaneous intramedullary hemorrhage: a report of a rare case repeated intramedullary hemorrhage with unknown etiology. Korean J Spine 2015;12:279-282. 